\section{The VVIQ and imagery-induced McCollough effects: An alternative analysis}

\section{J. P. HARRIS \\ Medical School, University of Bristol, Bristol, England}

In view of the recent discussion (Broetse \& Crassini, 1980, 1981; Finke, 1981) of the relationship between self-ratings of vividness of visual imagery and imagery-induced McCollough effects (Finke \& Schmidt, 1977, 1978; Kunen \& May, 1980; Kaufman, May, \& Kunen, 1981), it seemed worthwhile to look again at the original data (Finke \& Schmidt, 1978, Table 1) and examine how unequivocally they support the conclusion that subjects with visual imagery self-rated as more vivid report stronger imageryinduced McCollough effects (IIMEs). The reanalysis suggests an alternative view of the data, and of other effects which follow adaptation to partially imaginary stimuli.

Following an adapting session of "bar imagination" [subjects were shown two alternating fields, each a square matrix of 36 small black squares, one with a red and one a green background, and had to imagine that, say, the rows on red (and columns on green) formed horizontal (or vertical) bars], Finke and Schmidt presented achromatic split-field test gratings, and asked the subject to report which part of the pattern appeared most red (or least green). The number of forced choices (of a possible 20) whose direction corresponded to a negative McCollough effect was taken as a measure of the strength of the IIMEs. It was found that when subjects were divided into two groups of equal size on the basis of their self-ratings of vividness of imagery on the Vividness of Visual Imagery Questionnaire (VVIQ) of Marks (1973), the high-vividness group made significantly more negative McCollough-effect choices than the low-vividness group.

Before this analysis, data from nearly half the total subjects were discarded on the basis of their postexperimental reports of the strategies used in the forced choice test sessions. The "associative strategy" group $(n=4)$ reported "the deliberate association of color and orientation," while the "mixed strategy" group $(n=12)$ reported using the associative strategy on some trials but actually seeing colors on others. The "no-strategy" group $(n=19)$, whose data were used in the analysis, were those who reported that they "based their choices on perceived

The author thanks the MRC for support. His mailing address is: Brain and Perception Laboratory, Anatomy Department, Medical School, University Walk, Bristol BS8 ITD, England. color or guesses (or upon criteria totally unrelated to color-orientation pairing)." Broerse and Crassini (1981) suggest that the subjects' later rationalization of their behavior during the test session should not be taken unquestioningly as a reason for accepting or rejecting their data. In addition, it is arguable that this discarding of data does not produce a stringent test of the imagery hypothesis. It appears to be based on an assumption that a subject's strategy will be independent of the strength of any perceived colors on the black and white test stripes early in the McCollougheffect test session. But if the hypothesis is that people with vivid imagery will have relatively strong McCollough effects, they should fall naturally into the "no-strategy" group, and it seems wrong to eliminate on other grounds possible disconfirming data, from vivid imagers who might have reported few negative McCollough effects, or, indeed, confirming data from weak imagers who reported few McCollough effects but declared an inappropriate strategy. Some further analyses were therefore carried out on the complete set of data $(n=35)$ from all three strategy groups.

The total sample was first divided into two groups, of high and low vividness of imagery, on the basis of their published VVIQ scores, and the mean number of negative McCollough-effect judgments found for each group. (Since there was an odd number of subjects, the data of one subject from the middle of the VVIQ range were not used. In this particular analysis, the inclusion of the discarded data would have reduced the difference between the means). The mean number of McCollough-effect judgments from the high-vividness group was $9.8(\mathrm{SD}=8.4)$, and that from the low-vividness group was $8.7(\mathrm{SD}=6.1)$. These means were not significantly different $[\mathrm{t}(32)<$ $1, p>.05]$. The VVIQ scores and the number of negative McCollough-effect judgments were also ranked, and the correlation between the two sets of ranks was calculated. This correlation, though positive, was not significant (Spearman's rho $=.23$, $\mathrm{n}=35, \mathrm{p}>.05$ ). Thus, when the complete set of data is considered, the relationship between VVIQ score and number of negative McCollough-effect judgments becomes weak and statistically insignificant.

Is there another position from which to view these data? Inspection of the raw scores suggested that there might be a relationship between a subject's VVIQ score and the likelihood of his giving a number of McCollough-effect choices, whether positive or negative, which was well away from the chance level. This idea was tested by finding each subject's deviation-from-chance (DFC) score on the McCollough forced-choice task. Thus, a subject who made 10 negative McCollough-effect choices from a possible 
total of 20 would be given a score of 0 . A subject who reported either 18 or 2 negative McCollough effects would be given a score of 8 , and so on. Again, the total sample of subjects was divided into two groups on the basis of their VVIQ scores, data from one subject in the middle of the VVIQ range being discarded, which, if added, would have increased the difference between means for this particular analysis. The mean DFC scores were $7.7(\mathrm{SD}=2.6)$ for the high-vividness groups and $5.3(\mathrm{SD}=3.4)$ for the lowvividness group. These means were significantly different $[\mathrm{t}(32)=2.34, \mathrm{p}<.05]$. The DFC and VVIQ scores were also ranked, and the correlation between the two sets of ranks was found. Though small, it was significant (rho $=.37, \mathrm{n}=35, \mathrm{p}<.05$ ). These analyses suggest that, for the whole set of data, VVIQ scores are a better predictor of DFC scores than of negative McCollough-effect reports. In other words, whether their judgments correspond to "positive" or "negative" aftereffects, people with more vivid imagery, as reflected in their VVIQ scores, tend to be more consistent in their reports.

Why should this relationship be found? It is not easy to explain if one believes vividness of imagery to be important during adaptation, since the direction, as well as the strength of perceived colors, has then to be predicted. But suppose that vividness of imagery is important, not during adaptation, but during testing. The subject then has to guess what color he is expected to see. It would not be surprising if those subjects whose VVIQ scores suggest that their imagery was more vivid, or (as Gur and Hilgard, 1975 , remark of what it is that the VVIQ measures) more controllable, were more likely to imagine onto the test gratings the colors they guessed that they should see. Hence, it can be argued, the number of reports of McCollough effects, whether positive or negative, should deviate more from chance for highvividness subjects than for low. Moreover, it is possible that subjects whose imagery, as measured by the VVIQ, is most vivid may be those who are most susceptible to implicit suggestions by the experimenter that they should see colors on the test fields. Hoen (1978) found a low, but significant, positive correlation between scores on the VVIQ and Form C of the Stanford Hypnotic Susceptibility Scale. On this view, one would expect an "experimenter effect" in studies of IIMEs, which may be one of the reasons why Broerse and Crassini (1980) failed to find them.

Because no alternative to the imagery induction account of IIMEs, with its important implications for theories of sensory adaptation, has yet been published, it is perhaps worth seeing what else has to be added to this interpretation of Finke and Schmidt's (1978) results in order to account for the available evidence. First, some reason must be given for the finding (Finke \& Schmidt, 1977) that, after bar imagination, a majority of subjects guess that aftereffects should be negative and, after color imagination, that they should be positive. One possibility is that subjects learn from the afterimages of colored fields (seen during bar imagination between or at the edges of the stimuli) that aftereffects are negative. Since the afterimage of an achromatic grating (seen during color imagination) is an achromatic grating, its negative nature would be less obvious. This fits with Finke and Schmidt's report that subjects without experience of sensory adaptation guess that McCollough effects are positive. The above analysis of their VVIQ-related data suggests why subjects who report strong negative IIMEs after bar imagination also report strong positive IIMEs after color imagination. Second, an alternative account of the data of Kaufman, May, and Kunen (1981) and Kunen and May (1980) must be given. After bar imagination, these authors found negative IIMEs which they measured with a color-cancellation technique. Although this method is more convincing in this kind of experiment than that of counting forcedchoice responses, it seems possible that imagery of colors occurred during the test session and could have influenced the measurements in these experiments also, since it can elevate thresholds (Reeves, 1981). The further assumption must be made, then, that although they are not forced to report colors verbally, subjects nevertheless do imagine colors in the test session, perhaps because they were required to use imagery during the adapting session and are continuing to be "helpful." This idea might be tested in experiments in which imagery was manipulated during the test sessions, so that subjects were required to imagine colors that were the same as or complementary to the hypothetical IIMEs. If subjects were already imagining colors to give apparent IIMEs, instructions to imagine those colors should have little effect on color settings. One reason for taking this notion seriously is that interocularly transferred IIMEs are apparently negative (Kaufman, May, \& Kunen, 1981), and that IIMEs are contingent on the orientation of check edges, rather than of the fundamental spatial frequency of checkerboards, unless the to-be-imagined checkerboard is blurred during its preexperimental presentation to the subject (Kunen \& May, 1980). These effects are opposite to those found with external adapting stimuli and may reflect differences between vision and imagery, but, given the assumptions that subjects guess and imagine colors during testing and that (following bar imagination) imagined colors tend to be negative, the effects are in the direction which naive subjects would be likely to produce.

In summary, this note has suggested that the selection of subjects' data for analysis on the basis of their later descriptions of their strategies is questionable 
when the strength of their imagery is one of the variables under test. This selection is important for Finke and Schmidt's hypothesis that vivid imagers (according to self-report) give strong IIMEs, since without it the relationship evaporates. On the other hand, if all their data are included in the analysis, the VVIQ score is related to a forced-choice response score, whether corresponding to positive or negative IIMES, which is different from chance. On the basis of this result, it is suggested that vividness of imagery is important not during adaptation but during testing, when the subject may imagine the colors he believes should occur. Two other assumptions, that subjects guess that aftereffects should be negative after bar imagination and that they imagine what they think are appropriate colors during testing with color cancellation techniques, are necessary to explain available data. In the absence of alternative accounts of IIMEs, which are of great theoretical importance, these ideas seem worth investigation. Data which disproved them would clearly strengthen the hypothesis of imagery induction.

\section{REFERENCES}

Broense, J., \& Crassini, B. The influence of imagery ability on color aftereffects produced by physically present and imagined induction stimuli. Perception \& Psychophysics, 1980, 28, 560-568.
Broerse, J., \& Crassini, B. Misinterpretations of imageryinduced McCollough effects: A reply to Finke. Perception \& Psychophysics, 1981, 30, 96-98.

Finke, R. A. Interpretations of imagery-induced McCollough effects. Perception \& Psychophysics, 1981, 30, 94-95.

Finke, R. A., \& SchmidT, M. J. Orientation-specific color aftereffects following imagination. Journal of Experimental Psychology: Human Perception and Performance, 1977, 3, 599-606.

Finke, R. A., \& Schmidt, M. J. The quantitative measure of pattern representation in images using orientation-specific color aftereffects. Perception \& Psychophysics, 1978, 23, 515-520.

GUR, R. C., \& Hilgard, E. R. Visual imagery and the discrimination of differences between altered pictures simultaneously and successively presented. British Journal of Psychology, 1975, 66, 341-345.

HoEn, P. T. Effects of hypnotizability and visualizing ability on imagery-mediated learning. The International Journal of Clinical and Experimental Hypnosis, 1978, 26, $45-54$.

Kaufman, J. H., May, J. G., \& Kunen, S. Interocular transfer of orientation-contingent color aftereffects with internal and external adaptation. Perception \& Psychophysics, 1981, 30, 547-551.

KunEN, S., \& MAY, J. G. Spatial frequency content of visual imagery. Perception \& Psychophysics, 1980, 28, 555-559.

Marks, D. F. Visual imagery differences in the recall of pictures. British Journal of Psychology, 1973, 64, 17-24.

REEves, A. Visual imagery lowers sensitivity to hue-varying, but not to luminance-varying, visual stimuli. Perception \& Psychophysics, 1981, 29, 247-250.

(Manuscript received July 6, 1982;

accepted for publication July 6, 1982.) 